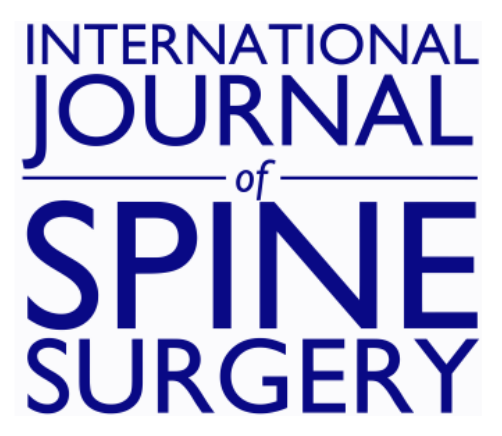

\title{
Outcomes of Same-Day Orthopedic Surgery: Are Spine Patients More Likely to Have Optimal Immediate Recovery From Outpatient Procedures?
}

Sara Naessig, Bhaveen H. Kapadia, Waleed Ahmad, Katherine Pierce, Shaleen Vira, Renaud Lafage, Virginie Lafage, Carl Paulino, Joshua Bell, Hamid Hassanzadeh, Michael Gerling, Themistocles Protopsaltis, Aaron Buckland, Bassel Diebo and Peter Passias

Int J Spine Surg 2021, 15 (2) 334-340

doi: https://doi.org/10.14444/8043

http://ijssurgery.com/content/15/2/334

This information is current as of April 26, 2023.

Email Alerts Receive free email-alerts when new articles cite this article. Sign up at: http://ijssurgery.com/alerts 


\title{
Outcomes of Same-Day Orthopedic Surgery: Are Spine Patients More Likely to Have Optimal Immediate Recovery From Outpatient Procedures?
}

\author{
SARA NAESSIG, BS, ${ }^{1}$ BHAVEEN H. KAPADIA, MD,${ }^{1}$ WALEED AHMAD, MS,${ }^{1}$ KATHERINE PIERCE, BS, ${ }^{1}$ \\ SHALEEN VIRA, MD, ${ }^{2}$ RENAUD LAFAGE, MS,${ }^{3}$ VIRGINIE LAFAGE, PHD,${ }^{3}$ CARL PAULINO, MD, ${ }^{4}$ \\ JOSHUA BELL, MD ${ }^{5}$ HAMID HASSANZADEH, MD ${ }^{5}$ MICHAEL GERLING, MD ${ }^{1}$ THEMISTOCLES \\ PROTOPSALTIS, MD ${ }^{1}$ AARON BUCKLAND, MD ${ }^{1}$ BASSEL DIEBO, MD,${ }^{4}$ PETER PASSIAS, MD $^{1}$ \\ ${ }^{I}$ Department of Orthopedics, NYU Langone Orthopedic Hospital, New York, New York, ${ }^{2}$ Department of Orthopedics, University of Texas Southwestern, Dallas, \\ Texas, ${ }^{3}$ Department of Orthopedics, Hospital for Special Surgery, New York, New York, ${ }^{4}$ Department of Orthopedics, SUNY Downstate Medical Center, \\ Brooklyn, New York, ${ }^{5}$ Department of Orthopedics, University of Virginia Charlottesville, Virginia
}

\begin{abstract}
Background: Spinal surgery is associated with an inherently elevated risk profile, and thus far there has been limited discussion about how these outpatient spine patients are benefiting from these same-day procedures against other typical outpatient orthopedic surgeries.

Methods: Orthopedic patients who received either inpatient or outpatient surgery were isolated in the American College of Surgeons National Surgical Quality of Improvement Program (2005-2016). Patients were stratified by type of orthopedic surgery received (spine, knee, ankle, shoulder, or hip). Mean comparisons and chi-squared tests assessed basic demographics. Perioperative complications were analyzed via regression analyses in regard to their principal inpatient or outpatient orthopedic surgery received.

Results: This study included 729480 surgical patients: $32.5 \%$ received spinal surgery, $36.5 \%$ knee, $24.1 \%$ hip, $4.9 \%$ shoulder, and $1.7 \%$ ankle. Of those who received a spinal procedure, $74.7 \%$ were inpatients (IN), and $25.3 \%$ were outpatients (OUT): knee: $96.1 \%$ IN, 3.9\% OUT; hip:98.9\% IN, 1.1\% OUT; ankle: $29 \%$ IN, $71 \%$ OUT; and shoulder: $52.6 \%$ IN, $47.6 \%$ OUT. Hip patients were the oldest, and knee patients had the highest body mass index out of the orthopedic groups $(P<.00)$. Spine IN patients experienced more complications than the other orthopedic groups and had the lowest OUT complications(both $P<.05$ ). This same trend of having higher IN complications than OUT complications was identified for hip, shoulder, and knee. However, ankle procedures had greater OUT procedure complications than IN $(P<.05)$. After controlling for age, body mass index, and Charlson Comorbidity Index, IN procedures, such as knee, hip, spine, and shoulder, were significantly associated with experiencing postoperative complications. From 2006 to 2016, IN and OUT surgeries were significantly different among complications experienced for all of the orthopedic groups $(P<.05)$ with complications decreasing for IN and OUT patients by 2016.

Conclusions: Over the past decade, spine surgery has decreased in complications for IN and OUT procedures along with IN/OUT knee, ankle, hip, and shoulder procedures, reflecting greater tolerance for risk in an outpatient setting.

Level of Evidence: 3.

Clinical Relevance: Despite the increase in riskier spine procedures, complications have decreased over the years. Surgeons should aim to continue to decrease inpatient spine complications to the level of other orthopedic surgeries.

Complications

Keywords: inpatient, outpatient, spine surgery, orthopedic surgery
\end{abstract}

\section{INTRODUCTION}

It is estimated that the incidence of spinal pathologies, such as degenerative disc disease, is on the rise in the United States, with a proportional increase in the number of procedures being performed. ${ }^{1,2}$ In recent decades, the number of lumbar procedures has more than doubled, with a similar surge seen in the number of cervical procedures being performed. ${ }^{3-5}$ These figures are of concern, as the disparity in healthcare expenditures continues to overwhelm hospital systems. Furthermore, with a shift in reimbursements toward bundle payments in the United States, there is further pressure on hospitals and surgeons to further reduce healthcare expenditures. $^{6}$ 
There has been much research into improving patient outcomes with the use of various methodologies, such as preoperative patient optimization, developing risk prediction models, and mitigating anticipated complications postoperatively. ${ }^{7-11}$ The synergistic effect of these modalities is an attempt to reduce patient morbidity and mortality as well as to reduce healthcare costs. An additional method of controlling costs is to transition from inpatient to outpatient procedures to reduce the high costs of hospital bed per day as well as supplies. ${ }^{12}$ The development of protocols for transitioning from outpatient to inpatient orthopedic procedures has seen tremendous success for various orthopedic procedures, such as total joint arthroplasty. ${ }^{13-18}$ These studies have found ambulatory surgery to be safe and effective and may potentially result in a reduction of nosocomial complications when compared to inpatient procedures.

Similarly, there has been a trend toward outpatient spinal procedures, many of which show it to be a feasible option to offset hospital costs without compromising patient safety. ${ }^{19-22}$ There is generally an inherently higher risk profile in patients undergoing spinal procedures when compared to other divisions of orthopedic surgeries. ${ }^{23}$ Although there has been much research comparing the safety and efficacy of inpatient versus outpatient spinal procedures, there is very limited (if any) literature comparing the outcomes of this transition to additional orthopedic procedures. Therefore, the purpose of this study was to assess outcomes and improvements in spinal inpatient and outpatient surgery when compared to other orthopedic procedures.

\section{METHODS}

\section{Study Design}

This is a retrospective cohort study of the American College of Surgeons National Surgical Quality of Improvement Program (ACS-NSQIP). The ACS-NSQIP tracks and audits 30-day perioperative outcomes of surgical patients across more than 400 medical institutions across the United States, and its sampling protocol has previously been described in the literature. ${ }^{7}$ The database includes demographic, comorbidity, surgical, and preoperative laboratory data as well as complication outcomes. This study includes data from the years 2005-2016. As the ACS-NSQIP data set is deidenti- fied and contains no geographic markers, this study was exempt from local institutional review board review. More information about the ACS-NSQIP program can be accessed at https://www.facs.org/ quality-programs/acs-nsqip/about.

\section{Inclusion Criteria}

All patients included in this study were adults (age $>18$ years) from the ACS-NSQIP database who underwent different types of orthopedic surgeries, such as spine, knee, ankle, shoulder, and hip, as defined via current procedural terminology.

\section{Statistical Analysis}

Descriptive statistics provided an overview of patient demographics, comorbidity burden (including a previously published NSQIP-modified Charlson Comorbidity Index, ${ }^{8}$ surgical factors, and complications. NSQIP is able to track whether a procedure is elective. As the vast majority of outpatient procedures are elective, to more clearly compare the impact of inpatient versus outpatient stay, only elective and nonemergency procedures were included. The NSQIP contains a variable specifically for ambulatory status defined as either inpatient or outpatient. We used this variable to separate inpatient cases from outpatient cases. Individual complications were analyzed among each orthopedic surgery both for its outpatient and for its inpatient components as well as other perioperative complications (return to the operating room, morbidity, and readmission). A subanalysis assessed complication rates when specific patient-specific demographics presented at baseline were controlled for with binary logistic regressions. Descriptive, mean comparison, and binary logistic regression analyses were conducted in SPSS (version 23.0, Armonk, SPSS Inc, New York).

\section{RESULTS \\ Cohort Overview}

A total of 729480 surgical patients were included: $32.5 \%$ received spinal surgery, $36.5 \%$ knee, $24.1 \%$ hip, $4.9 \%$ shoulder, and $1.7 \%$ ankle. By basic demographics, hip patients were the oldest, and knee patients had the highest body mass index out of the orthopedic groups (Table $1 ; P<.001$ ). The top spine procedures were fusions $(53.2 \%)$, decompressions $(54.7 \%)$, laminotomies $(15.4 \%)$, and laminectomies $(6.5 \%)$. 
Table 1. Basic demographics by orthopedic surgery.

\begin{tabular}{|c|c|c|c|c|c|c|c|}
\hline & Spine & Knee & Hip & Ankle & Shoulder & Neck & $P$ Value \\
\hline Age, mean $\pm \mathrm{SD}, \mathrm{y}$ & $57 \pm 14.3$ & $65.6 \pm 10.9$ & $65.6 \pm 12.3$ & $47 \pm 15.7$ & $61.1 \pm 14.6$ & $39.7 \pm 16.4$ & $<.001$ \\
\hline Gender (female), \% & 48.1 & 60.6 & 56.2 & 42.2 & 47.0 & 49.2 & $>.05$ \\
\hline Body mass index, mean $\pm \mathrm{SD}, \mathrm{kg} / \mathrm{m}^{2}$ & $30.2 \pm 13$ & $32.8 \pm 7.1$ & $29.5 \pm 6.6$ & $30.6 \pm 6.8$ & $30.7 \pm 6.8$ & $26.4 \pm 6.2$ & $<.001$ \\
\hline Charlson Comorbidity Index, mean $\pm \mathrm{SD}$ & $1.04 \pm 0.95$ & $1.05 \pm 0.87$ & $1.0 \pm 0.91$ & $0.68 \pm 0.88$ & $1.01 \pm 0.93$ & $0.87 \pm 1.44$ & $>.05$ \\
\hline
\end{tabular}

\section{Breaking Down by Ambulatory Setting}

Table 2 displays the breakdown of inpatients (IN) and outpatients (OUT) for these orthopedic surgeries. Spine IN patients experienced more complications than the other orthopedic procedures (14\% vs $10.2 \% ; P<.001)$; however, spine OUT procedures had a lower complication rate $(6.3 \%$ vs $10.8 \% ; P<$ $.001)$. A breakdown of type of complications by specific orthopedic procedures is shown in Table 3. Calculated ratios were analyzed for the rate of complication of switching from inpatient to outpatient. Switching spine patients from inpatient to outpatient had an $18.1 \%$ decrease in the number of postoperative complications. This switch in ambulatory setting was not identified to be beneficial for other orthopedic surgeries with a $35.5 \%$ increase in postoperative complications.

\section{Trends of Orthopedic Surgeries by Setting}

From 2006 to 2016, IN spine procedure complications, decreased while other orthopedic surgeries increased. However, during this time, OUT spine complications increased, while other orthopedic complications decreased, as shown in Table 4.

\section{Complication Rate: Spine Versus All Orthopedic Surgeries}

Compared to other orthopedic procedures, spine patients had the greatest overall complication rate $(12.1 \%$ vs $10.3 \% ; P<.001)$. More specifically, spine patients had greater rates of reoperation $(3 \%$ vs $2 \%)$, sepsis $(0.7 \%$ vs $0.4 \%)$, urinary tract infections $(1.3 \%$ vs $1.1 \%)$, and deep surgical site infections $(0.6 \%$ vs $0.3 \%$; all $P<.001)$. Table 3 breaks down specific postoperative complications

Table 2. Orthopedic surgery by ambulatory setting (\%).

\begin{tabular}{lcr}
\hline & Inpatient & Outpatient \\
\hline Spine $(\mathrm{n}=237$ 391) & 74.7 & 25.3 \\
Knee (n=265 875) & 96.1 & 3.9 \\
Hip $(\mathrm{n}=175666)$ & 98.9 & 1.1 \\
Ankle $(\mathrm{n}=12412)$ & 28.4 & 71.6 \\
Shoulder $(\mathrm{n}=35692)$ & 52.3 & 47.7 \\
Neck $(\mathrm{n}=1865)$ & 90.4 & 9.5 \\
\hline
\end{tabular}

by orthopedic specialties when compared to spine procedures. Multivariate analysis identified postoperative complications associated with various orthopedic surgeries. Table 5 displays significant IN procedures that are independent predictors for developing a complication. After controlling for age and Charlson Comorbidity Index, IN spine patients were significantly associated with postoperative complications (odds ratio [OR]: 0.42; confidence interval $[\mathrm{CI}]: 0.40-0.43 ; P<.001)$ as well as IN ankle patients (OR: 0.54; CI 0.48-0.61; $P<$ .001). OUT knee patients were identified to be significantly associated with this outcome (OR: 1.1; CI: $1.08-1.2 ; P<.001$; Table 5).

\section{Specific Spine Procedures by Complications}

Spine procedures, such as fusions and laminectomies, when compared against other orthopedic surgeries, had greater rates of complications, as shown in Table 6. However, decompressions were significantly different only for sepsis complications $(0.5 \%$ vs $0.4 \%)$ and had a lower overall complication rate $(10 \%$ vs $10.3 \%$; all $P<.05)$. When looking at arthroscopic rotator cuff repair against cervical spine fusions, the latter had greater rates of readmission, reoperation, surgical site infection, sepsis, and overall complications (all $P<.05$; Table $6)$. A similar trend was also seen when comparing total hip arthroplasties and lumbar spine procedures.

\section{DISCUSSION}

There has been a marked increase in the number of spine procedures being performed in the United States as well as worldwide, which is estimated to have more than doubled in the previous decades. $^{3-5,24-26}$ Additionally, there has been a concomitant net increase in healthcare costs for these procedures, with estimates of over $\$ 35$ billion from 2001 to 2010 in the United States. ${ }^{27,28}$ One method of reducing this expenditure would be to remove the most obviously exorbitant cost of an inpatient hospital bed, which is estimated to be over $\$ 5000$ 
Table 3. Complications by orthopedic surgery $(\%){ }^{a}$

\begin{tabular}{|c|c|c|c|c|c|c|c|c|c|c|}
\hline & \multicolumn{2}{|c|}{ Spine } & \multicolumn{2}{|c|}{ Knee } & \multicolumn{2}{|c|}{ Hip } & \multicolumn{2}{|c|}{ Ankle } & \multicolumn{2}{|c|}{ Shoulder } \\
\hline & IN & OUT & IN & OUT & IN & OUT & IN & OUT & IN & Out \\
\hline $\mathrm{N}$ & 30583 & 5726 & 255629 & 10246 & 173744 & 1922 & 3527 & 8885 & 18673 & 17019 \\
\hline Total complications & $14.0 *$ & $6.3^{*}$ & 10.0 & 10.0 & 10.1 & 10.1 & $19.3 *$ & $11.2 *$ & $12.8^{*}$ & $11.2 *$ \\
\hline Readmission & $58.2 *$ & $55.8^{*}$ & $59.2 *$ & $72.4^{*}$ & $53.1^{*}$ & $74.3^{*}$ & 69.3 & 68.7 & 65.4 & 66.2 \\
\hline Return to operating room & $2.3 *$ & $0.9^{*}$ & $1.0^{*}$ & $0.8^{*}$ & $1.6^{*}$ & $0.8^{*}$ & $2.1 *$ & $0.4^{*}$ & $1.0^{*}$ & $0.4^{*}$ \\
\hline Stroke & $0.2 *$ & $0.0^{*}$ & 0.1 & 0.0 & 0.2 & 0.1 & 0.0 & 0.0 & $0.1 *$ & $0.0^{*}$ \\
\hline Urinary tract infection & $1.6^{*}$ & $0.3^{*}$ & $0.9^{*}$ & $0.4^{*}$ & 1.6 & 1.0 & $0.7 *$ & $0.2 *$ & $0.8^{*}$ & $0.2^{*}$ \\
\hline Acute renal failure & $0.1 *$ & $0.0^{*}$ & $0.1^{*}$ & $0.0^{*}$ & 0.1 & 0.1 & $0.1 *$ & $0.0 *$ & $0.1 *$ & 0.0 \\
\hline Cardiac arrest & $0.2 *$ & $0.0 *$ & $0.1^{*}$ & $0.0^{*}$ & 0.2 & 0.2 & 0.0 & 0.0 & $0.1 *$ & $0.0^{*}$ \\
\hline Myocardial infarction & $0.3^{*}$ & $0.1 *$ & $0.2^{*}$ & $0.0^{*}$ & 0.4 & 0.3 & $0.2 *$ & $0.0^{*}$ & $0.2^{*}$ & 0.0 \\
\hline Bleeding transfusions & $8.4^{*}$ & $0.2 *$ & $7.0^{*}$ & $0.7 *$ & $12.9^{*}$ & $5.7 *$ & $2.4^{*}$ & $0.0 *$ & $4.1 *$ & $0.02 *$ \\
\hline Deep vein thrombosis & $0.8^{*}$ & $0.2 \% *$ & $0.9^{*}$ & $0.5^{*}$ & 0.5 & 0.4 & 0.2 & 0.5 & $0.4^{*}$ & $0.1^{*}$ \\
\hline Sepsis & $0.9 *$ & $0.2 *$ & $0.4^{*}$ & $0.1^{*}$ & 0.5 & 0.3 & $0.5^{*}$ & $0.1 *$ & $0.3^{*}$ & $0.1^{*}$ \\
\hline
\end{tabular}

${ }^{a}$ Asterisk indicates significant difference between IN and OUT group for that particular orthopedic surgery.

per day, and performing procedures on an outpatient basis. $^{12}$ As a transition takes place toward ambulatory cervical and lumbar procedures, it is important to understand their safety when compared to inpatient procedures as well as in comparison to other orthopedic procedures. ${ }^{29-31}$ Our study found that spine procedures were associated with higher complications in the inpatient setting and lower complications in outpatient cases when compared to other orthopedic procedures.

Although there are no level I or II studies assessing the safety of inpatient versus outpatient spine procedures, there has been some literature demonstrating it to be a safe medium for surgery. ${ }^{13,15-18,20,21,32,33}$ A systematic review of 56 articles evaluating ambulatory spine surgery found that a majority of studies reported no differences in peri- and postoperative complications, readmissions, and reoperations when compared to inpatient cases. ${ }^{19,33,34}$ These findings indicate that outpatient spine cases may be a safe alternative to the traditional inpatient cases. Furthermore, a metaanalysis of studies evaluating cervical and lumbar spine surgeries, with a total of 370,195 patients,

Table 4. Trends of complications of spine versus orthopedic surgeries (\%).

\begin{tabular}{lcccccc}
\hline & $\mathbf{2 0 0 6}$ & $\mathbf{2 0 0 8}$ & $\mathbf{2 0 1 0}$ & $\mathbf{2 0 1 2}$ & $\mathbf{2 0 1 4}$ & $\mathbf{2 0 1 6}$ \\
\hline $\begin{array}{l}\text { Inpatient } \\
\quad \text { Spine }\end{array}$ & 39.9 & 35.3 & 33.8 & 34 & 36.9 & 36.5 \\
$\quad \begin{array}{l}\text { Other orthopedic } \\
\text { Outpatient }\end{array}$ & 60.1 & 64.7 & 66.2 & 66 & 63.1 & 63.5 \\
$\quad$ Spine & 33.3 & 42.3 & 45.1 & 64 & 70 & - \\
$\quad$ Other orthopedic & 66.7 & 57.7 & 54.9 & 36 & 30 & - \\
$\begin{array}{l}\text { Complications by inpatient } \\
\quad \text { Spine }\end{array}$ & 43.8 & 36.4 & 33.8 & 34.0 & 37.0 & 37.2 \\
$\quad$ Other orthopedic & 56.2 & 63.6 & 66.2 & 66.0 & 63.0 & 62.8 \\
$\begin{array}{l}\text { Complications by outpatient } \\
\quad \text { Spine }\end{array}$ & 53.5 & 43.5 & 45.1 & 64.0 & 70.0 & 68.7 \\
$\quad$ Other orthopedic & 46.5 & 56.5 & 54.9 & 36.0 & 30.0 & 31.3 \\
\hline
\end{tabular}

demonstrated that outpatient procedures had significantly lower overall costs when compared to inpatient procedures. ${ }^{22}$ When substratified by age, the study showed that costs for younger patients were markedly lower than procedures for older patients ( $\$ 555$ vs $\$ 7290$, respectively). This discrepancy in costs is likely attributed to generally higher comorbidity and therefore risk in older patients undergoing surgery. ${ }^{35}$

Similar trends have been observed in other outpatient orthopedic procedures when compared to inpatient procedures, which showed similar postoperative complications and lower overall costs. A study by Darrith et $\mathrm{l}^{16}$ evaluated 243 consecutive patients undergoing outpatient arthroplasty procedures and compared them to a matched cohort undergoing inpatient arthroplasty and tracked them for 90 days. The authors effectively demonstrated no significant differences when comparing readmission rates, major and minor complications, reoper-

Table 5. Independent predictors of postoperative complications.

\begin{tabular}{lcc}
\hline & $\begin{array}{c}\text { Beta } \\
\text { (Confidence Interval) }\end{array}$ & $\boldsymbol{P}$ Value \\
\hline $\begin{array}{c}\text { Postoperative complications } \\
\text { (no controls) }\end{array}$ & & \\
Orthopedic surgery & & \\
Spine & & $<.001$ \\
Knee & $0.4(0.39-0.42)$ & .9 \\
Hip & $1.0(0.93-1.07)$ & .9 \\
Ankle & $1.0(0.86-1.1)$ & $<.001$ \\
Shoulder & $0.53(0.47-0.59)$ & $<.001$ \\
Postoperative complication & $0.86(0.81-0.92)$ & \\
$\quad$ controlling for age and & & $<.001$ \\
$\quad$ Charlson Comorbidity Index & & .001 \\
Spine & $0.42(0.40-0.43)$ & .10 \\
Knee & $1.1(1.08-1.2)$ & \\
Hip & $1.1(1.3-1.32)$ & .17 \\
Ankle & $0.54(0.48-0.61)$ & \\
Shoulder & $0.9(0.88-1.0)$ & \\
\hline
\end{tabular}


Table 6. Complication rate for specific spine procedures against other orthopedic procedures (\%). ${ }^{a}$

\begin{tabular}{|c|c|c|c|c|}
\hline \multicolumn{5}{|c|}{ Spine Versus All Orthopedic Surgeries } \\
\hline Parameter & Fusion & Decompression & Laminectomy & All Orthopedic \\
\hline Total complications & $13.7 *$ & $10.0^{*}$ & $13.7 *$ & 10.3 \\
\hline Reoperation & $3.4 *$ & 2.0 & $3.8^{*}$ & 2.0 \\
\hline Readmission & 52.7 & 54.0 & $45.3^{*}$ & 58.4 \\
\hline Sepsis & $0.9^{*}$ & $0.5^{*}$ & $1.5^{*}$ & 0.4 \\
\hline Surgical site infection & $0.7^{*}$ & 0.3 & $0.8^{*}$ & 0.3 \\
\hline Urinary tract infection & $1.6^{*}$ & 0.8 & $2.5^{*}$ & 1.1 \\
\hline Pulmonary embolism & 0.5 & 0.3 & $0.7^{*}$ & 0.5 \\
\hline \multicolumn{5}{|c|}{ Spine Versus Specific Orthopedic Surgeries } \\
\hline Parameter & Cervical & Rotator Cuff Repair & Lumbar Fusion & Hip Arthroplasty \\
\hline Total complications & $28.6^{*}$ & $10.2^{*}$ & $10.4^{*}$ & 8.4 \\
\hline Reoperation & $2.1 *$ & $0.8^{*}$ & $3.7 *$ & $2.1 *$ \\
\hline Readmission & $71.0^{*}$ & $62.4^{*}$ & 0.2 & 0.5 \\
\hline Sepsis & $0.5^{*}$ & $0.2^{*}$ & $0.7 *$ & $0.3^{*}$ \\
\hline Surgical site infection & $0.3^{*}$ & $0.1 *$ & $0.5^{*}$ & $0.3^{*}$ \\
\hline Urinary tract infection & 0.0 & 0.0 & $1.5^{*}$ & $1.1 *$ \\
\hline Pulmonary embolism & 0.0 & 0.0 & $0.6^{*}$ & $0.3^{*}$ \\
\hline
\end{tabular}

${ }^{a}$ Asterisk indicates significance when compared against orthopedics overall or specific orthopedic surgery.

ations, emergency room visits, and unplanned clinic visits between the 2 cohorts.

However, some studies have even showed better overall outcomes of outpatient procedures when compared to inpatient cases. For example, a study by Huntley et $\mathrm{al}^{36}$ reviewed 7672 patients undergoing foot and ankle surgery using the ACS-NSQIP database. When compared to outpatient cases, the authors found that patients undergoing inpatient procedures had significantly higher surgical $(8.6 \%$ vs $2.0 \%)$ and medical complications $(16.9 \%$ vs $1.7 \%)$. These findings appear promising for surgeons considering outpatient procedures as a safe alternative to inpatient cases; however, additional findings in this study highlight the importance of underlying patient demographics and comorbidities as the true deciding components of overall outcomes. Unsurprisingly, the inpatient group represented a higher-risk population when compared to the outpatient group, with a higher mean age, more smokers, poorer functional status, and, most important, a disproportionately higher prevalence of every comorbidity. This is similar to the findings of our study, as patients who had higher comorbidities, which occur often in the spine patient population, had more complications when compared to other orthopedic procedures. This strongly suggests the role and importance of careful patient selection when considering outpatient procedures as well as a potentially existing bias inherent in current studies evaluating these 2 modalities.
There were some limitations in our study, particularly the retrospective nature. Furthermore, there are several inherent known limitations with the use of the NSQIP database, with a reliance on the accuracy of ICD-9 and CPT codes to identify patient outcomes. ${ }^{37}$ There may have been coding inconsistencies that potentially resulted in an underor overrepresentation of effects and outcomes. Additionally, as many complications and reoperations can occur for up to 1 year from the index procedure, many of these may be missed since the database allows patient tracking for only 30 days postoperatively. In spite of some of these limitations, we feel that the use of a large database allows us to have adequate power to potentially overcome biases and coding errors to effectively assess the main question of our study.

The pressures to transition from inpatient to outpatient spine procedures appear to be multifactorial, influenced also by changes in reimbursement as bundled payment models. ${ }^{6}$ Furthermore, as the Centers for Medicare and Medicaid Services transitions from inpatient-only procedures to outpatient, insurance companies are also denying preauthorization for inpatient stays following surgery, which may translate to spine surgeries. ${ }^{32}$ As outpatient procedures may be riskier due to less postoperative observation, the advent of additional patient optimization strategies and selection is ever important. Additionally, the use of ambulatory procedures has resulted in the development and refinement of minimally invasive techniques in an 
attempt to further mitigate and reduce complications. ${ }^{29,38}$

\section{CONCLUSIONS}

The results of our study found that inpatient spine surgery patients had higher complications than outpatient when compared to other orthopedic cases. It is possible that inpatient procedures carry with them an inherent sense of safety and comfort with constant medical observation, possibly resulting in less attention to minute patient risk factors and therefore operating on higher-risk patients, which is in contrast to outpatient procedures potentially requiring a more careful evaluation, more aggressive intervention, and improved risk mitigation. Further studies are needed not only to normalize outcomes within a surgical specialty but also to explore variations in subspecialty patient risks as an important means of understanding the multifactorial components contributing to patient complications and hospital costs following any orthopedic procedure, both inpatient and outpatient.

\section{REFERENCES}

1. Disease and Injury Incidence and Prevalence Collaborators. Global, regional, and national incidence, prevalence, and years lived with disability for 354 diseases and injuries for 195 countries and territories, 1990-2017: a systematic analysis for the Global Burden of Disease Study 2017. Lancet. 2018;392(10159):1789-1858.

2. Oichi T, Taniguchi Y, Oshima Y, Tanaka S, Saito T. Pathomechanism of intervertebral disc degeneration. JOR Spine. 2020;3(1). doi:10.1002/jsp2.1076

3. Rajaee SS, Bae HW, Kanim LE, Delamarter RB. Spinal fusion in the United States: analysis of trends from 1998 to 2008. Spine (Phila Pa 1976). 2012;37(1):67-76.

4. Yoshihara H, Yoneoka D. National trends in the surgical treatment for lumbar degenerative disc disease: United States, 2000 to 2009. Spine J. 2015;15(2):265-271.

5. Stein BE, Hassanzadeh H, Jain A, Lemma MA, Cohen DB, Kebaish KM. Changing trends in cervical spine fusions in patients with rheumatoid arthritis. Spine (Phila Pa 1976). 2014;39(15):1178-1182.

6. Tanenbaum JE, Miller JA, Alentado VJ, et al. Insurance status and reportable quality metrics in the cervical spine fusion population. Spine J. 2017;17(1):62-69.

7. Yagi M, Hosogane N, Fujita N, et al. Surgical risk stratification based on preoperative risk factors in adult spinal deformity. Spine J. 2018;19(5):816-826.

8. Kyrola K, Kautiainen H, Pekkanen L, Makela P, Kiviranta I, Hakkinen A. Long-term clinical and radiographic outcomes and patient satisfaction after adult spinal deformity correction. Scand J Surg. 2018. https://doi.org/10.1177/ 1457496918812201
9. Leven DM, Lee NJ, Kothari P, et al. Frailty Index is a significant predictor of complications and mortality after surgery for adult spinal deformity. Spine (Phila Pa 1976). 2016;41(23):E1394-E1401.

10. Shah K, Kothari M, Nene A. Role of frailty scoring in the assessment of perioperative mortality in surgical management of tuberculous spondylodiscitis in the elderly. Global Spine J. 2018;8(7):698-702.

11. Ali R, Schwalb JM, Nerenz DR, Antoine HJ, Rubinfeld I. Use of the modified frailty index to predict 30-day morbidity and mortality from spine surgery. $J$ Neurosurg Spine. 2016;25(4):537-541.

12. Ogink PT, Karhade AV, Thio Q, et al. Development of a machine learning algorithm predicting discharge placement after surgery for spondylolisthesis. Eur Spine J. 2019;28(8):1775-1782.

13. Basques BA, Tetreault MW, Della Valle CJ. Same-day discharge compared with inpatient hospitalization following hip and knee arthroplasty. J Bone Jt Surg Am. 2017;99(23):19691977.

14. Bosch LC, Bala A, Denduluri SK, et al. Reimbursement and complications in outpatient vs inpatient unicompartmental arthroplasty. J Arthroplasty. 2020;35(suppl 6):S86-S91.

15. Crawford DC, Li CS, Sprague S, Bhandari M. Clinical and cost implications of inpatient versus outpatient orthopedic surgeries: a systematic review of the published literature. Orthop Rev (Pavia). 2015;7(4). doi:10.4081/or.2015.6177

16. Darrith B, Frisch NB, Tetreault MW, Fice MP, Culvern CN, Della Valle CJ. Inpatient versus outpatient arthroplasty: a single-surgeon, matched cohort analysis of 90-day complications. J Arthroplasty. 2019;34(2):221-227.

17. Greenky MR, Wang W, Ponzio DY, Courtney PM. Total hip arthroplasty and the Medicare inpatient-only list: an analysis of complications in Medicare-aged patients undergoing outpatient surgery. J Arthroplasty. 2019;34(6):1250-1254.

18. Nwankwo CD, Dutton P, Merriman JA, Gajudo G, Gill K, Hatch J. Outpatient total shoulder arthroplasty does not increase the 90-day risk of complications compared with inpatient surgery in prescreened patients. Orthopedics. 2018;41(4):e563-e568.

19. DelSole EM, Makanji HS, Kurd MF. Current trends in ambulatory spine surgery: a systematic review. J Spine Surg. 2019;5(suppl 2):S124-S132.

20. Lee MJ, Kalfas I, Holmer H, Skelly A. Outpatient surgery in the cervical spine: is it safe? Evid Based Spine Care J. 2014;5(2):101-111.

21. Hirsch BP, Khechen B, Patel DV, Cardinal KL, Guntin JA, Singh K. Safety and efficacy of revision minimally invasive lumbar decompression in the ambulatory setting. Spine (Phila Pa 1976). 2019;44(8):E494-E499.

22. Mundell BF, Gates MJ, Kerezoudis P, et al. Does patient selection account for the perceived cost savings in outpatient spine surgery? A meta-analysis of current evidence and analysis from an administrative database. $J$ Neurosurg Spine. 2018;29(6):687-695.

23. Walsh MT. Improving outcomes in ambulatory anesthesia by identifying high risk patients. Curr Opin Anaesthesiol. 2018;31(6):659-666.

24. Cancelliere $\mathrm{C}$, Wong JJ, $\mathrm{Yu} \mathrm{H}$, et al. Postsurgical rehabilitation for adults with low back pain with or without radiculopathy who were treated surgically: protocol for a mixed 
studies systematic review. BMJ Open. 2020;10(3). doi:10.1136/ bmjopen-2020-036817

25. Kim CH, Chung CK, Kim MJ, et al. Increased volume of lumbar surgeries for herniated intervertebral disc disease and cost-effectiveness analysis: a nationwide cohort study. Spine (Phila Pa 1976). 2018;43(8):585-593.

26. Sivasubramaniam V, Patel HC, Ozdemir BA, Papadopoulos MC. Trends in hospital admissions and surgical procedures for degenerative lumbar spine disease in England: a 15-year time-series study. BMJ Open. 2015;5(12). doi:10.1136/ bmjopen-2015-009011

27. Goz V, Weinreb JH, McCarthy I, Schwab F, Lafage V, Errico TJ. Perioperative complications and mortality after spinal fusions: analysis of trends and risk factors. Spine (Phila Pa 1976). 2013;38(22):1970-1976.

28. Harada GK, Basques BA, Samartzis D, Goldberg EJ, Colman M, An HS. Development and validation of a novel scoring tool for predicting facility discharge after elective posterior lumbar fusion. Spine J. 2020;20(10):1629-1637.

29. Baird EO, Brietzke SC, Weinberg AD, et al. Ambulatory spine surgery: a survey study. Global Spine J. 2014;4(3):157160.

30. Baird EO, Egorova NN, McAnany SJ, Qureshi SA, Hecht AC, Cho SK. National trends in outpatient surgical treatment of degenerative cervical spine disease. Global Spine J. 2014;4(3):143-150.

31. Deyo JC, Smith BH, Biola H, et al. Reducing high-risk medication use through pharmacist-led interventions in an outpatient setting. J Am Pharm Assoc (2003). 2020;60(4):e86e92.

32. Edwards PK, Milles JL, Stambough JB, Barnes CL, Mears SC. Inpatient versus outpatient total knee arthroplasty. $J$ Knee Surg. 2019;32(8):730-735.

33. Segal DN, Wilson JM, Staley C, Yoon ST. Outpatient and inpatient single-level cervical total disc replacement: a comparison of 30-day outcomes. Spine (Phila Pa 1976). 2019;44(1):79-83.

34. Bovonratwet P, Fu MC, Tyagi V, Ondeck NT, Albert TJ, Grauer JN. Safety of outpatient single-level cervical total disc replacement: a propensity-matched multi-institutional study. Spine (Phila Pa 1976). 2019;44(9):E530-E538.

35. Miller EK, Neuman BJ, Jain A, et al. An assessment of frailty as a tool for risk stratification in adult spinal deformity surgery. Neurosurg Focus. 2017;43(6). doi:10.3171/2017.10. FOCUS17472

36. Huntley SR, McGee AS, Johnson JL, et al. Outcomes of inpatient versus outpatient elective foot and ankle surgery. Cureus. 2019;11(2):e4058.

37. Alluri RK, Leland H, Heckmann N. Surgical research using national databases. Ann Transl Med. 2016;4(20). doi:10. 21037/atm.2016.10.49

38. Adogwa O, Parker SL, Bydon A, Cheng J, McGirt MJ. Comparative effectiveness of minimally invasive versus open transforaminal lumbar interbody fusion: 2-year assessment of narcotic use, return to work, disability, and quality of life. $J$ Spinal Disord Tech. 2011;24(8):479-484.

Disclosures and COI: Institutional review board approval was waived, as this study uses a deidentified nationwide database.

Corresponding Author: Peter Passias, MD, New York Spine Institute, NYU Langone Orthopedic Hospital, Departments of Neurologic and Orthopaedic Surgery, Division of Spine, 301 East 17th Street, New York, NY 10003. Phone: (516) 357-8777; Fax: (516) 357-0087; Email: Peter. Passias@nyumc.org.

Published 16 April 2021

This manuscript is generously published free of charge by ISASS, the International Society for the Advancement of Spine Surgery. Copyright $\odot 2021$ ISASS. To see more or order reprints or permissions, see http://ijssurgery.com. 